\title{
Electromagnetic field prediction - scattered and total field approaches
}

\author{
Chandan Bhat*, Karteekeya Sastry ${ }^{\dagger}$, Raffaele Solimene ${ }^{\ddagger}$ and Uday K. Khankhoje* \\ *Electrical Engineering, Indian Institute of Technology Madras, Chennai, India. \\ C. Bhat (e-mail: ee16d209@smail.iitm.ac.in), U. K. Khankhoje (corresponding author, email: uday@ee.iitm.ac.in). \\ ${ }^{\dagger}$ California Institute of Technology, Pasadena, CA 91125, USA (e-mail: karteekdhara98@gmail.com). \\ ‡Universita della Campania Luigi Vanvitelli, Italy (e-mail: raffaele.solimene@unicampania.it).
}

\begin{abstract}
We summarize recent approaches to estimate electromagnetic fields in an arbitrary two-dimensional scattering domain. Only an estimate of the objects' spatial support in the scattering environment is required in our approach. Using the Huygens' principle and compressive sensing, the tangential electromagnetic fields on the scatterers' surface are estimated by making few field measurements in the scattering domain. Based on the field measurements, two inverse formulations are discussed in the paper: (a) scattered field inverse (SFI) and (b) total field inverse (TFI). For an indoor scattering domain with three scattering objects, the total electric field is recovered with an accuracy of $92 \%$, and the incident field is recovered with an accuracy of $96 \%$ when the number of measurements is 0.3 times the number of unknowns in which the problem is formulated.
\end{abstract}

Index Terms-Compressive Sensing, Electromagnetic Fields, Sensor Placement, Inverse Scattering.

\section{INTRODUCTION}

In our recent works [1], [2], we have developed techniques to predict the electromagnetic (EM) fields in an arbitrary scattering environment. Such problems have many applications, such as in $\mathrm{WiFi}$ access point provisioning. It is known that existing solutions based on ray-tracing approaches [3], [4] work in the high frequency regime but fail to capture effects such as diffraction around object corners, multiple reflections [5, Fig. 3], and suffer from high errors in near-field estimation. In this paper, we present an overview of our techniques that overcome these limitations based on the surface integral (SI) method that models the wave-matter interactions exactly.

Most of the inverse problems in EM require the information of the scattered field [6], which implies that every experiment should have two sets of measurements (a) total field and (b) incident field. To overcome this, we present a general technique that maps the EM field in any given scattering environment using only the total field measurements. The inverse technique requires a knowledge of only the approximate spatial support of the scatterers. We assume that the region in-between the scatterers is homogeneous. In our earlier work [1], we assumed that the incident field was known, and used random sensing locations to get field measurements. In our follow-up work [2], we dropped the requirement of incident field knowledge. Additionally, we also explored optimal sensor placement strategies that gave better results than when the measurements were taken at random locations.

\section{Problem Overview}

Region 0

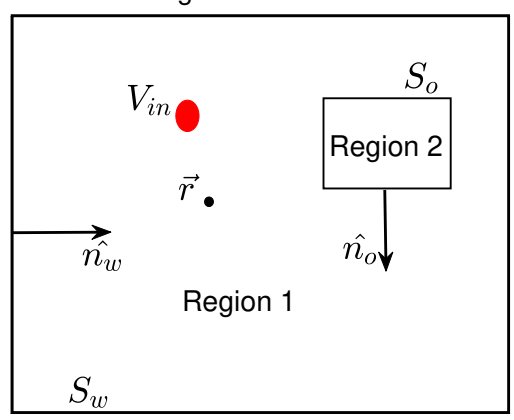

Fig. 1. Problem schematic: $S_{w}$ is the inner surface of the enclosing wall and $S_{o}$ is the outer surface of a non-magnetic scatterer. $V_{i n}$ denotes the volume enclosing the source. $\hat{n}_{w}$ and $\hat{n}_{o}$ are the normals to the scattering surfaces of the wall and object respectively.

The electromagnetic field at any location $\vec{r}$ in Region 1 of the two dimensional (2D) schematic shown in Fig .1 can be obtained using the Huygens' principle [7], which states that the scattered fields can be expressed in terms of the freespace Green's function and the tangential electric and magnetic fields on the scattering surfaces. For a transverse magnetic (TM) polarization, the total field at any point $\vec{r}$ in Region 1 is expressed as:

$$
\begin{aligned}
& \phi(\vec{r})=\phi_{\text {in }}(\vec{r}) \\
& -\oint_{S_{w}}\left[g\left(\vec{r}, \vec{r}^{\prime}\right) \nabla^{\prime} \phi_{w}\left(\vec{r}^{\prime}\right)-\phi_{w}\left(\vec{r}^{\prime}\right) \nabla^{\prime} g\left(\vec{r}, \vec{r}^{\prime}\right)\right] \cdot \hat{n}_{w} d l^{\prime} \\
& -\oint_{S_{o}}\left[g\left(\vec{r}, \vec{r}^{\prime}\right) \nabla^{\prime} \phi_{o}\left(\vec{r}^{\prime}\right)-\phi_{o}\left(\vec{r}^{\prime}\right) \nabla^{\prime} g\left(\vec{r}, \vec{r}^{\prime}\right)\right] \cdot \hat{n}_{o} d l^{\prime},
\end{aligned}
$$

where $\phi(\vec{r})$ is the total electric field at $\vec{r}, \phi_{\text {in }}(\vec{r})$ is the incident field, $g\left(\vec{r}, \vec{r}^{\prime}\right)=-\frac{j}{4} H_{0}^{(2)}\left(k_{0}\left|\vec{r}-\vec{r}^{\prime}\right|\right)$ is the 2D free space Green's function ( $k_{0}$ is the free space wavenumber) and $\hat{n}_{w}, \hat{n}_{o}$ is the unit normal from the surfaces $S_{w}$ and $S_{o}$ into Region 1. $\phi_{o}, \phi_{w}$ are the tangential electric fields on the object and wall respectively, and $\nabla \phi_{o} . \hat{n}_{o}, \nabla \phi_{w} . \hat{n}_{w}$ are proportional to the tangential magnetic fields on the object and wall, respectively. 
In Eq. (1) both the tangential electric and magnetic fields over the scattering surfaces $S_{w}, S_{o}$ are used to determine field at any location, which is redundant as per the Uniqueness theorem [8]. To eliminate this redundancy, we use the Extinction theorem [7] for Region 1, which establishes the following relation between tangential electric and magnetic fields

$$
\begin{gathered}
\phi_{\text {in }}(\vec{r})-\oint_{S_{w}}\left[g\left(\vec{r}, \vec{r}^{\prime}\right) \nabla^{\prime} \phi_{w}\left(\vec{r}^{\prime}\right)-\phi_{w}\left(\vec{r}^{\prime}\right) \nabla^{\prime} g\left(\vec{r}, \vec{r}^{\prime}\right)\right] \cdot \hat{n}_{w} d l^{\prime} \\
-\oint_{S_{o}}\left[g\left(\vec{r}, \vec{r}^{\prime}\right) \nabla^{\prime} \phi_{o}\left(\vec{r}^{\prime}\right)-\phi_{o}\left(\vec{r}^{\prime}\right) \nabla^{\prime} g\left(\vec{r}, \vec{r}^{\prime}\right)\right] \cdot \hat{n}_{o} d l^{\prime} \\
=0, \quad \vec{r} \in\left\{S_{w}, S_{o}\right\}
\end{gathered}
$$

\section{A. Scattered Field and Total Field formulation}

From the Huygens' principle (Eq. (1)), the field at any location $\vec{r}$ can be obtained if the tangential fields $\left(\nabla \phi_{w}, \phi_{w}, \nabla \phi_{o}, \phi_{o}\right)$ on the scattering surfaces and the source function $\phi_{i n}(\vec{r})$ are known. We formulate two different problems to estimate the field at any point $\vec{r}$ in Region 1 ,

1) Scattered Field Inverse (SFI): Here, the incident field is known, while the tangential field is to be determined [1].

2) Total Field Inverse (TFI): Here, both the incident field and tangential fields are unknown [2].

Depending on the problem (either SFI or TFI), the unknowns are expanded in terms of known basis functions. For example, in TFI the incident field is expanded using Graf's addition theorem [9, Section 9.1] and tangential fields are expanded in the pulse basis. The unknown coefficients of the corresponding basis functions are estimated using the field measurements at various locations.

\section{B. Defining Data and State equations}

After expanding the unknowns in the appropriate basis functions, the discretized versions of Eq. (1) and (2) are called the Data and State equations respectively. The Data and State equations for SFI and TFI are as follows:

1) SFI-Data equation: $A_{d} \boldsymbol{x}=\boldsymbol{b}_{\boldsymbol{d}}+\boldsymbol{\nu}$ where $A_{d} \in \mathbf{C}^{M \times N}, \boldsymbol{x} \in \mathbf{C}^{N}$ and $\boldsymbol{b}_{\boldsymbol{d}} \in \mathbf{C}^{M}, \boldsymbol{b}_{\boldsymbol{d}}$ contains the scattered field measurements at $M$ locations, $N=$ $2\left(N_{o}+N_{w}\right) . N_{o}, N_{w}$ are the number of discretized segments of the surface $S_{o}$ and $S_{w}$ respectively. The scattered field measurements are corrupted by noise given in $\nu$. The elements of the matrix $A_{d}$ are computed as per the relation in [1, Eq. (9)]

2) SFI - State equation: $A_{s} \boldsymbol{x}=\boldsymbol{b}_{\boldsymbol{s}}$, where $A_{s} \in \mathbf{C}^{\frac{N}{2} \times N}$, and $\boldsymbol{b}_{\boldsymbol{s}} \in \mathbf{C}^{\frac{N}{2}}, \boldsymbol{b}_{\boldsymbol{s}}$ contains the incident field measurements on surface of the scatterers $\left(S_{o}, S_{w}\right)$.

\section{3) TFI - Data equation: $A_{d} \boldsymbol{x}=\boldsymbol{b}_{\boldsymbol{d}}+\boldsymbol{\nu}$} where $A_{d} \in \mathbf{C}^{M \times\left(N+N_{i}\right)}, \boldsymbol{x} \in \mathbf{C}^{N+N_{i}}$ and $\boldsymbol{b}_{\boldsymbol{d}} \in \mathbf{C}^{M}, \boldsymbol{b}_{\boldsymbol{d}}$ contains the total field measurements at $M$ locations. The total field measurements are corrupted by noise given in $\nu$.

4) TFI - State equation: $A_{s} \boldsymbol{x}=0$, where $A_{s} \in$ $\mathbf{C}^{\frac{N}{2} \times\left(N+N_{i}\right)}$, and $0 \in \mathbf{R}^{\frac{N}{2}} \cdot N_{i}$ is the number of unknown coefficients for the incident field expansion.

\section{Optimal sensor placement for field measurements}

One of the important steps in the inverse problem is to measure the field at several spatial locations. In [1] the field measurements were taken at random locations in the scattering domain (Region 1 in Fig. 1). In our followup work [2], we investigated the problem of optimal sensor placement; that of choosing the best $M$ locations out of $P$. There are $\left(\begin{array}{c}P \\ M\end{array}\right)$ possibilities to place $M$ sensors out of $P$ locations. We use a greedy algorithm called Maximum Projection on Minimum Eigenspace (MPME) [10] that finds the best $M$ sampling locations out of all possible locations, $P$.

\section{Outline of our method}

To predict the EM field at any given location, we follow the steps given below.

1) Measure the total/scattered fields (TFI/SFI) at $M$ locations in Region 1 in Fig. 1.

2) Solve an optimization problem to find the unknown field coefficients, $\boldsymbol{x}$, that satisfy both the Data and the State equations.

3) Substitute the estimated coefficients in the Huygens' principle to predict the total/scattered field at any desired location in Region 1.

\section{Solution Methodology}

We now discuss the algorithm used to solve the unknown vector $\boldsymbol{x}$ in both the SFI and TFI formulations. Within the framework of the Subspace optimization method (SOM) [11], the vector $\boldsymbol{x}$ is split into two orthogonal spaces (a) Major space component- which is recovered from the measured data, and (b) Minor space component- the tangential fields are sparse in the discrete Fourier transform (DFT) domain, hence principles of compressive sensing (CS) is used to recover this component.

The Major space component, $\boldsymbol{x}_{1}$, is determined by employing the truncated singular value decomposition (SVD) solution to the Data equation, given by:

$$
\boldsymbol{x}_{1}=\sum_{i=1}^{L_{0}}\left(\frac{\boldsymbol{u}_{i}^{H} \boldsymbol{b}}{\sigma_{i}}\right) \boldsymbol{v}_{i}
$$

where, $\boldsymbol{b}=\boldsymbol{b}_{\boldsymbol{d}}+\boldsymbol{\nu},\left\{\boldsymbol{u}_{i}\right\}_{i=1}^{M},\left\{\boldsymbol{v}_{i}\right\}_{i=1}^{N}$ are the left and right singular vectors of $A_{d}$ respectively, and $\left\{\sigma_{i}\right\}_{i=1}^{M}$ are the singular values of $A_{d}$ arranged in descending order. The value of $L_{0}$ is chosen using the Morozov Discrepancy Principle [12]. The procedure for estimation of the major space component $\boldsymbol{x}_{1}$ in both SFI and TFI is the same.

For determining the Minor space component, $\boldsymbol{x}_{2}$, we solve the following optimization problem:

1) SFI:

$$
\begin{array}{cc}
\underset{\boldsymbol{x}_{2}}{\operatorname{minimize}} & \left\|\mathcal{M}\left(\boldsymbol{x}_{1}+\boldsymbol{x}_{2}\right)\right\|_{1} \\
\text { subject to } & \left\|A_{d}\left(\boldsymbol{x}_{1}+\boldsymbol{x}_{2}\right)-\boldsymbol{b}\right\|_{2} \leq \epsilon \\
& \left\|A_{s}\left(\boldsymbol{x}_{1}+\boldsymbol{x}_{2}\right)-\boldsymbol{b}_{\boldsymbol{s}}\right\|_{2} \leq \eta
\end{array}
$$


2) TFI:

$$
\begin{array}{cc}
\underset{\boldsymbol{x}_{2}}{\operatorname{minimize}} & \left\|\mathcal{M} I_{1}\left(\boldsymbol{x}_{1}+\boldsymbol{x}_{2}\right)\right\|_{1}+t\left\|I_{2}\left(\boldsymbol{x}_{1}+\boldsymbol{x}_{2}\right)\right\|_{2} \\
\text { subject to } & \left\|A_{d}\left(\boldsymbol{x}_{1}+\boldsymbol{x}_{2}\right)-\boldsymbol{b}\right\|_{2} \leq \epsilon \\
& \left\|A_{s}\left(\boldsymbol{x}_{1}+\boldsymbol{x}_{2}\right)\right\|_{2} \leq \eta
\end{array}
$$

where $\mathcal{M}=\mathcal{F}$ or $\mathcal{D}$ is the discrete fourier transform (DFT) or the discrete cosine transform (DCT) basis respectively, $b$ is scattered field measurements for SFI and total field measurements for TFI. $I_{1} \in \mathbf{R}^{N \times\left(N+N_{i}\right)}$ and $I_{2} \in \mathbf{R}^{N_{\mathrm{i}} \times\left(N+N_{i}\right)}$ are blockwise identity matrices which when multiplied with a $N+N_{i}$ dimensional vector return the first $N$ and last $N_{\mathrm{i}}$ elements of that vector respectively, and $t$ is a regularization parameter; $\epsilon$ is the square root of the noise variance, $\eta$ is an estimate of the discretization error in the state equation. We solve the above problems using the CVX package [13], [14].

\section{NUMERICAL RESULTS}

In this Section, we present the results of various numerical simulations to evaluate the performance of the proposed method. All the simulations are programmed in MATLAB $\mathrm{R} 2019 \mathrm{~b}$ on a $2.4 \mathrm{GHz}$ Quad-Core Intel Core i5 processor, using 8GB RAM.

\section{A. Simulation setup and generating the synthetic measure-} ments

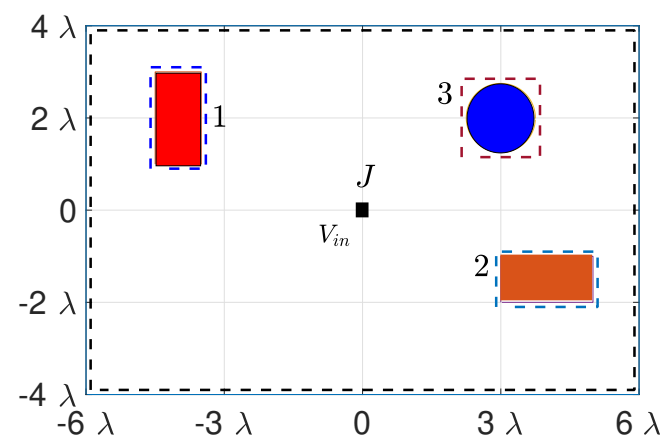

Fig. 2. The schematic of a $12 \lambda \times 8 \lambda$ simulation domain, where $\lambda$ is the wavelength of the incident wave in free space. The domain includes a wall, 3 objects and a source. Object 1 and 2 are rectangles of sides $\lambda \times 2 \lambda$, and $2 \lambda \times \lambda$ and object 3 is a circle with radius $0.75 \lambda$. The relative permittivities of respective objects are $\epsilon_{r 1}=1.7-2.1 j, \epsilon_{r 2}=2.6-1.1 j, \epsilon_{r 3}=1.8-0.9 j$ and the relative permittivity of the wall is $\epsilon_{r w}=3.7-2.1 j$. The objects 1,2 , and 3 are centered at $(-4 \lambda, 2 \lambda),(4 \lambda,-1.5 \lambda)$, and $(3 \lambda, 2 \lambda)$. The source is placed within a $\lambda / 4 \times \lambda / 4$ square that is centered at origin. The exact shape of the objects are indicated by the filled regions and the dotted contours are the approximate object contours used in the problem solution.

The simulation setup is chosen such that it considers the scattering in the indoor setup ( Fig. 2). The relative permittivities of the scattering objects are complex so that it mimics the real world objects. We generate the synthetic (total field and scattered field) measurements using a Boundary Integral [7] solver with pulse basis function and delta testing function. The frequency of operation is $1.5 \mathrm{GHz}$, and the forward solver is discretized at $\lambda / 40$. The problem can be scaled to have more objects and also a larger simulation domain. The exact shape and permittivities of the objects are only required to generate the synthetic measurements.

Any general source function can be considered for generating the synthetic field data. We define the source current $J$ placed in a region $V_{i n}$ (see Fig. 2) as follows:

$$
J(x, y)= \begin{cases}I_{0}\left(x^{2}+y^{2}-x+y\right) & (x, y) \in V_{\text {in }} \\ 0 & \text { else }\end{cases}
$$

where $I_{0}$ is a normalization constant such that the magnitude of the incident field at a radius of $10 \lambda$ is approximately unity. A $6 \times 6$ Gauss Legendre quadrature rule in $2 \mathrm{D}$ is used to calculate the true incident field for generating synthetic measurements.

The inverse formulation uses the synthetically generated total/scattered field by the forward solver and also approximate spatial support of the objects that are seen as dotted contours in Fig. 2.

\section{B. Inverse solver}

For inverse formulation, the approximate geometry of the object is discretized at $\lambda / 5$, which provides an optimal balance between the computational cost and accuracy. The source function is confined in a square of side $\lambda / 4$, and the number of coefficients considered with respect to incident field expansion is $N_{i}=5$ [2]. The total number of unknown variables for the inverse formulation are: $2 \times(197+34+34+32)=592$ for SFI and 597 for TFI. The Data matrix is constructed from the obtained sampling locations. The sampling locations are generated with two different methods (a) random sampling scheme and (b) optimal samples obtained from MPME. We measure the scattered field and total field at the sampling locations depending on the method of inverse solver i.e. SFI or TFI respectively. The measurements are corrupted by additive white gaussian noise (AWGN) with a $25 \mathrm{~dB}$ signal to noise ratio (SNR). The field prediction is done for two different number of measurements (also called as sampling rate). Sampling rate (SR) is defined as the ratio of number of measurements to the total number of unknowns. The major part of the solution Eq. (3) is obtained using the Morozov discrepancy principle [12]. For the minor part of the solution we solve Eqs. $(4,5)$ with the DCT as the transformation matrix.

\section{Field prediction comparison : optimal sampling scheme $(M P M E)$ vs Random sampling}

$\boldsymbol{x}$ is estimated by making few field measurements based on the locations obtained by different sampling schemes, and the estimated tangential field components are substituted in Eq. (1) to predict the field over the entire simulation domain $(12 \lambda \times 8 \lambda)$. The reconstructed and true fields are compared to obtain various error metrics: the relative error in the field prediction over a grid in Region 1 is indicated by $\Delta G$, and the relative error in the incident field estimation is given by $\Delta I$. We do not consider the locations inside the scatterers and locations that are very close to the scattering surfaces (distance less than $\lambda / 10)$. 
We compare the performance of both the sampling schemes for the SFI and TFI formulation. In both the methods the optimal sampling scheme reconstructs the fields with higher accuracy for the same sampling rate. Fig. 3 shows the 2D error comparison for SR of 0.3 .

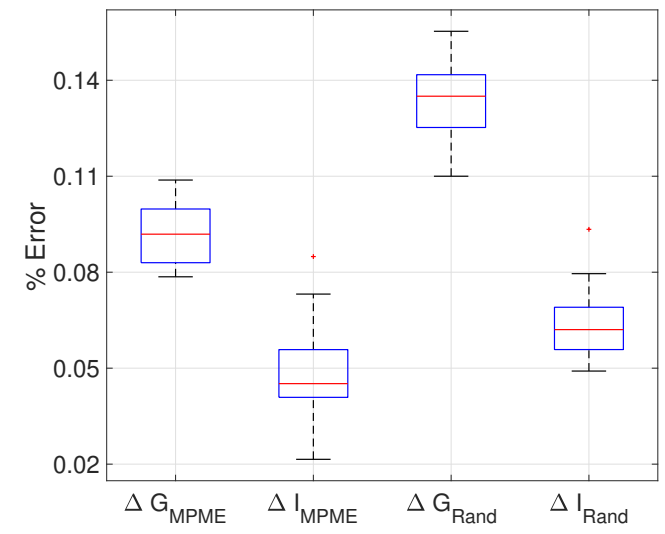

Fig. 3. Box plot error comparison for MPME sampling scheme and Random sampling scheme for TFI at $0.3 \mathrm{SR}$ (179 measurements). $\triangle G_{M P M E}$ and $\Delta G_{\text {Rand }}$ are total field error for MPME and random sampling scheme. $\triangle I_{M P M E}$ and $\triangle I_{\text {Rand }}$ are incident field error for MPME and random sampling scheme. The error plotted is for 50 monte carlo iterations. Red line is the average of the error over 50 iterations, the bottom and top of each box are the $25^{t h}$ and $75^{t h}$ percentiles of the error distribution.

The reconstruction of fields is done in two ways because of the difference in the amount of information considered for the simulations. Total field formulation (TFI) measures only the total field without the knowledge of the incident fields, hence we estimate the total field. And the scattered field formulation (SFI) uses both the incident field as well as total field information so we estimate the scattered fields. The reconstructed $2 \mathrm{D}$ total field using the TFI method is shown in Fig. 4. The measurements are done using sampling locations obtained by MPME samples at 0.3 SR. Fig. 5 shows the sampling locations obtained from MPME (optimal sampling) and random sampling scheme.

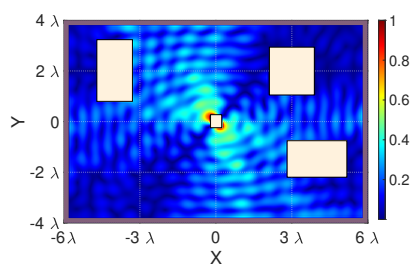

(a) True

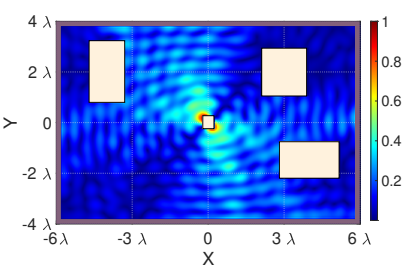

(b) Estimated
Fig. 4. Normalized magnitudes of (a) True and (b) estimated total fields by making 178 measurements using MPME (0.3 SR, $25 \mathrm{~dB}$ SNR). Colorbar shows the field magnitude in $V / m$. The total field is estimated for TFI formulation with $9 \%$ error.

\section{CONCLUSION}

In this paper, we have given an overview of two algorithms that have been recently developed by us to predict the

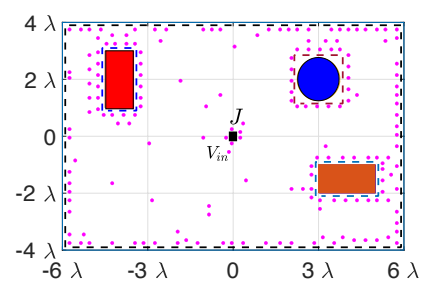

(a) MPME (b) Random

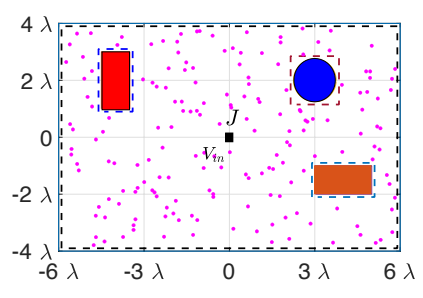

Fig. 5. Sampling locations obtained from (a) MPME (Optimal sampling) scheme (b) random sampling scheme. The 'filled' dots are the sampling locations where field measurements are taken. The number of sampling locations are 179.

EM field over any given scattering environments by making either, (a) scattered field measurements, or (b) total field measurements. We compare the reconstruction performance of both the methods for random sampling (RS) scheme and optimal sampling locations obtained by MPME. Using optimal sampling locations, the average reconstruction error is $8 \%$ for a sampling rate (SR) of 0.3 and an SNR of $25 \mathrm{~dB}$ compared with $14 \%$ error for RS at the same noise level. The reconstruction error with optimal locations shows a good improvement compared with the random sampling scheme at $0.3 \mathrm{SR}$, but as the SR increases the improvement becomes less significant. The TFI formulation does not assume any information on the source function and the incident field is estimated with a of $3 \%$.

\section{REFERENCES}

[1] C. Bhat, K. Sastry, and U. K. Khankhoje, "Compressive sensing approaches for the prediction of scattered electromagnetic fields," JOSA $A$, vol. 37, no. 7, pp. 1166-1174, 2020.

[2] K. Sastry, C. Bhat, R. Solimene, and U. K. Khankhoje, "Electromagnetic field imaging in arbitrary scattering environments," 2020, (Under Review).

[3] A. Toscano, F. Bilotti, and L. Vegni, "Fast ray-tracing technique for electromagnetic field prediction in mobile communications," IEEE transactions on magnetics, vol. 39, no. 3, pp. 1238-1241, 2003.

[4] V. Degli-Esposti, D. Guiducci, A. de'Marsi, P. Azzi, and F. Fuschini, "An advanced field prediction model including diffuse scattering," IEEE Transactions on Antennas and Propagation, vol. 52, no. 7, pp. 1717 1728, 2004.

[5] K. A. Remley, H. R. Anderson, and A. Weisshar, "Improving the accuracy of ray-tracing techniques for indoor propagation modeling," IEEE transactions on vehicular technology, vol. 49, no. 6, pp. 23502358, 2000.

[6] K. Belkebir and M. Saillard, "Testing inversion algorithms against experimental data," Inverse problems, vol. 17, no. 6, p. 1565, 2001.

[7] W. C. Chew, Waves and fields in inhomogeneous media. IEEE press, 1995.

[8] J. Kong, Electromagnetic Wave Theory. A Wiley-Interscience publication, Wiley, 1986.

[9] M. Abramowitz and I. A. Stegun, Handbook of mathematical functions: with formulas, graphs, and mathematical tables, vol. 55. Courier Corporation, 1965.

[10] C. Jiang, Y. C. Soh, and H. Li, "Sensor placement by maximal projection on minimum eigenspace for linear inverse problems," IEEE Transactions on Signal Processing, vol. 64, no. 21, pp. 5595-5610, 2016.

[11] X. Chen, "Subspace-based optimization method for solving inversescattering problems," IEEE Transactions on Geoscience and Remote Sensing, vol. 48, no. 1, pp. 42-49, 2009. 
[12] Y. Sanghvi, Y. N. G. B. Kalepu, and U. Khankhoje, "Embedding deep learning in inverse scattering problems," IEEE Transactions on Computational Imaging, 2019.

[13] M. Grant and S. Boyd, "CVX: Matlab software for disciplined convex programming, version 2.1." http://cvxr.com/cvx, Mar. 2014.

[14] M. Grant and S. Boyd, "Graph implementations for nonsmooth convex programs," in Recent Advances in Learning and Control (V. Blondel, S. Boyd, and H. Kimura, eds.), Lecture Notes in Control and Information Sciences, pp. 95-110, Springer-Verlag Limited, 2008. http://stanford. edu/ boyd/graph_dcp.html.

This paper's copyright is held by the author(s). It is published in these proceedings and included in any archive such as IEEE Xplore under the license granted by the "Agreement Granting EurAAP Rights Related to 